\title{
Blind Phase Noise Estimation for CO-OFDM Transmissions
}

\author{
Son Thai Le, Student member, IEEE, Paul A. Haigh, Member, IEEE, Andrew D. Ellis \\ and Sergei K. Turitsyn \\ (Invited paper)
}

\begin{abstract}
In this paper, we discuss in detail the performance of different blind phase noise estimation schemes for coherent optical orthogonal frequency division multiplexing transmissions. We first derive a general model of such systems with phase noise. Based on this model, the phase cycle slip probability in blind phase noise estimation is calculated. For blind phase tracking, we present and discuss the implementation of feedback loop and digital phase tracking. We then analyze in detail the performance of a decision-direct-free blind scheme, in which only three test phases are required for phase noise compensation. We show that the decision-direct-free blind scheme is transparent to QAM formats and can provide a similar performance to the conventional blind phase search employing 16 test phases. We also propose two novel cost functions to further reduce the complexity of this scheme.
\end{abstract}

Index Terms - Coherent, phase noise, orthogonal frequency division multiplexing, phase estimation.

\section{INTRODUCTION}

C oherent optical orthogonal frequency division multiplexing (CO-OFDM) has attracted a lot of attention recently as a promising candidate for long-haul optical communication systems because of its inherent spectral efficiency (SE) and excellent tolerance towards residual linear fiber impairments [1]. However, compared to single carrier system, traditional CO-OFDM system with cyclic prefix (CP) has relatively long symbol duration (tens of ns), and therefore is sensitive to laser phase noise, which introduces both common phase error (CPE) and intercarrier interference (ICI) [2]. To increase the system's SE and reduce the impact of ICI, reduced-guard-interval (RGI) CO-OFDM transmission scheme has been proposed in [3], where the overhead due to $\mathrm{CP}$ can be significantly reduced as fiber chromatic dispersion is compensated for within the receiver rather than using the CP. In this case, the impact of laser phase noise on the system performance is dominated by the CPE rather than ICI [3].

The CPE in RGI CO-OFDM transmission can be effectively compensated by inserting pilot subcarriers across the OFDM band [4]. However, this technique reduces the system spectral

Manuscript received June 01, 2015. S. T. Le, A. D. Ellis and S. K. Turitsyn are with Aston Institute of Photonic Technologies (AIPT), Aston Triangle, Birmingham, B4 7ET, UK (corresponding author phone: +44(0)744-702-4009; e-mail: let1@aston.ac.uk). P. A. Haigh is with Bristol University. Copyright (c) 2015 IEEE. Personal use of this material is permitted. However, permission to use this material for any other purposes must be obtained from the IEEE by sending a request to pubs-permissions@iee.org. efficiency as the overhead due to pilot subcarriers can be up to $10 \%$ due to the small number of subcarriers $(<200)$ in RGI CO-OFDM transmissions. To address this issue, a datadependent pilot-aided (quasi-pilot-aided) technique was introduced in $[5,6]$ to reduce the overhead due to pilot subcarriers by a factor of 2 , without compromising the performance. In addition, a pre-emphasized pilot subcarrier technique was considered in [7], significantly reducing the pilot subcarrier overhead at a cost of increasing the pilot subcarrier power overhead (up to 10\%). However, it is still desirable to remove completely the overhead due to pilot subcarriers to maximize the system's SE and power consumption.

Blind phase noise estimation for CO-OFDM with a small number of subcarriers (up to 200) has attracted a lot of attention recently [8-10]. In [8], a blind phase noise estimation (PNE) method based on a decision-directed (DD) algorithm has been considered for CO-OFDM. A major advantage of DD-based PNE scheme is that it is compatible with any modulation format. However, this technique suffers significantly from error propagation, and thus, it cannot be applied directly in the presence of a large laser phase noise. In [11] the concept of blind phase search (BPS) was proposed, which can be applied effectively without suffering from error propagation. However, the BPS technique also relies on DD (to estimate the mean-square-error) and a large number test phases (16 to 32) are required to achieve good performance and thus, being too complex for practical implementation.

To address the drawbacks of both BPS and DD-based PNE schemes, a novel decision-directed-free (DDF) blind PNE technique has been proposed and experimentally demonstrated recently in [12]. This DDF blind PNE scheme provides several advantages. Firstly, it offers a high performance without decision feedback, avoiding error propagation. Secondly, it requires only three test phases and thus significantly reduces the computational complexity in comparison to BPS. Finally, DDF blind scheme is also transparent to QAM formats.

In this paper, we provide a detailed analysis of DDF blind PNC technique and investigate its performance for high order modulation formats up to 64QAM. We also propose here two novel cost functions to further reduce the complexity of DDF blind PNC scheme.

The remainder of the paper is organized as follows. The system model is described in Section II. In Section III blind phase tracking with feedback loop (FL) and digital phase tracking (DPT) are discussed. In Sections IV-VI, the performances and complexities of DDF blind PNE with 


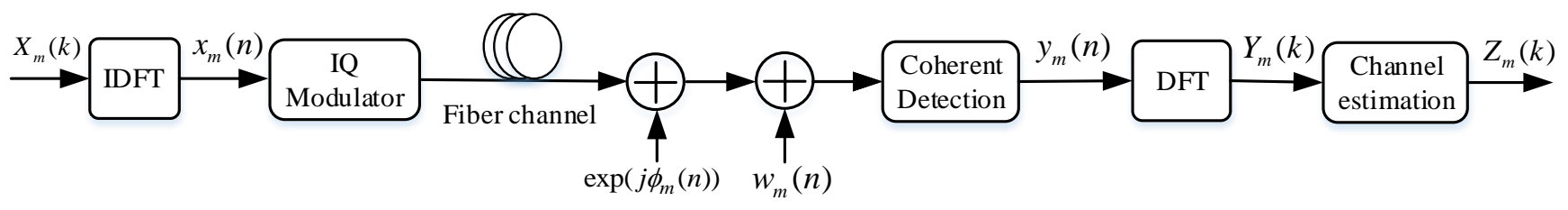

Fig. 1. Block diagram of the CO-OFDM system with laser phase noise and nonlinear phase noise acquired during optical fiber transmission

different cost functions are analyzed and compared with pilotaided (PA) and BPS techniques. Section VII concludes the paper.

\section{SYSTEM MODEL}

In this section, we introduce the models of laser phase noise, fiber channel, and a general RGI CO-OFDM system with phase noise (Fig. 1). For simplicity, the impact of fiber nonlinearity is not considered. In addition, we assume that perfect FFT window synchronization and frequency offset compensation are achieved. Furthermore, we consider a baseband system with single polarization signals noting that the analysis can be readily extended to passband systems with dual polarized signals without any difficulties.

\section{A. Phase noise model}

The laser phase noise $\phi(t)$, generated at both transmitter and receiver, can be modeled as a continuous Brownian motion or a Wiener process with zero mean and variance $\sigma^{2}=2 \pi \beta t$, where $\beta$ denotes the combined laser linewidth, i.e., frequency spacing between 3-dB points of the Lorentzian power spectral density function $[13,14]$. The discrete-time model of the laser phase noise on the $n^{\text {th }}$ sample of the $m^{\text {th }}$ OFDM symbol can be expressed as

$$
\phi_{m}(n)=\phi_{m-1}(N-1)+\sum_{i=-N_{C P}}^{n} u\left(m\left(N+N_{C P}\right)+i\right)
$$

where $u(i)$ represents the independently incremental movement of phase noise at time instant $i$ and is Gaussian distributed with zero mean and variance $\sigma^{2}=2 \pi \beta d t$, where $d t$ is the sampling time, $N$ and $N_{C P}$ are the DFT length and cyclic prefix length, respectively.

\section{B. Fiber channel model}

We consider here only dispersion-compensation-free fiber transmission links (highly dispersed channels). In addition, we assume here that the fiber loss is perfectly compensated using optical amplifiers such as erbium doped fiber amplifiers or Raman-based amplifiers. As a result, the fiber channel model can be simply expressed in the frequency domain as

$$
H(z, \omega)=\exp \left(-j \frac{D \lambda^{2} z}{4 \pi c} \omega^{2}\right)
$$

where $\omega$ is the angular frequency, $z$ is the transmission distance, $D$ is the fiber chromatic dispersion parameter, $c$ is the speed of light and $\lambda$ is the carrier wavelength.

\section{CO-OFDM system model}

In OFDM systems, the $m^{\text {th }}$ symbol in the time domain is generated from the modulated data in the frequency domain using the inverse discrete Fourier transform (IDFT) operation as follows

$$
x_{m}(n)=\frac{1}{\sqrt{N}} \sum_{k=0}^{N-1} X_{m}(k) \exp \left(\frac{j 2 \pi n k}{N}\right)
$$

where the samples $n$ range from - $N_{C P}$ to $N-1$.

Due to the ASE noise, chromatic dispersion and phase noise the received OFDM signal can be written as

$$
y_{m}(n)=\exp \left(j \phi_{m}(n)\right)\left[x_{m}(n) \otimes F^{-1}\left(H_{m}(k)\right)\right]+w_{m}(n)
$$

where $\otimes$ and $F^{-1}(\cdot)$ denote the circular convolution and IDFT, respectively, while $w_{m}(\mathrm{n})$ indicates the total ASE noise generated from inline optical amplifiers, $H_{m}(k)$ is the channel gain in the frequency domain of the $k^{\text {th }}$ subcarrier defined as (2). We assume here that the intersymbol interference is absent, after taking the DFT operation we obtain

$$
Y_{m}(k)=X_{m}(k) H_{m}(k) I_{m}(0)+I C I_{m}(k)+W_{m}(k),
$$

where the intercarrier interference $I C I_{\mathrm{m}}(k)$ is defined as

$$
I C I_{m}(k)=\sum_{l=0, l \neq k}^{N-1} X_{m}(l) H_{m}(l) I_{m}(l-k),
$$

where $I_{\mathrm{m}}(k)$ is given by

$$
I_{m}(k)=\frac{1}{N} \sum_{n=0}^{N-1} \exp \left(j \phi_{m}(n)\right) \exp \left(-j \frac{2 \pi k n}{N}\right)
$$

From (6) and (2) the system model can be written as

$$
Y_{m}(k)=X_{m}(k) H_{m}(k) I_{m}(0)+\varepsilon_{m}(k),
$$

where the accumulated noise is defined as

$$
\varepsilon_{m}(k)=I C I_{m}(k)+W_{m}(k)
$$

The channel response in CO-OFDM systems can be obtained by periodically inserting training sequences. By assuming perfect channel estimation, the recovered data after equalization can be expressed as

$$
Z_{m}(k)=Y_{m}(k) / H_{m}(k)
$$

As a result, we have

$$
Z_{m}(k)=X_{m}(k) I_{m}(0)+\chi_{m}(k),
$$


where the equalization-enhanced phase noise (EEPN) is defined as

$$
\chi_{m}(k)=\varepsilon_{m}(k) / H_{m}(k)
$$

Due to the complicated interplay among laser phase noise, and fiber dispersion, most of ICI compensation techniques developed for OFDM systems in linear (radio or open space) channels [15-17] cannot be effectively applied for CO-OFDM transmission systems. In addition, except the pulse shaping approach [18], ICI compensation techniques are usually highly complex and thus, cannot be applied effectively in high speed CO-OFDM transmission systems. A recent study [7] has shown that the EEPN term in the expression (11) can be simply treated as a zero mean Gaussian noise.

In the expression (11), $I_{m}(0)$ is usually referred to the common phase error as it corresponds to the time-average of the laser phase noise over the $m^{\text {th }}$ OFDM symbol

$$
I_{m}(0)=\frac{1}{N} \sum_{n=0}^{N-1} \exp \left(j \phi_{m}(n)\right) \approx \exp (j \Phi(m)),
$$

where $\Phi(m)$ is the CPE of the $\mathrm{m}^{\text {th }}$ OFDM symbol defined as:

$$
\Phi(m)=\frac{1}{N} \sum_{n=0}^{N-1} \phi_{m}(n)
$$

Finally, the system model can be simplified as

$$
Z_{m}(k)=X_{m}(k) \exp (j \Phi(m))+\chi_{m}(k)
$$

In this case, the CO-OFDM system model with phase noise converges to the linear OFDM system model with constant phase offset [2]. As a result, even though we focus our discussions in this paper on RGI CO-OFDM systems for fiber link, all the techniques and results presented here are fully applicable for traditional OFDM systems in radio frequency domain.

\section{BLIND PHASE NOISE TRACKING}

In CO-OFDM systems, in general, blind PNE can be implemented without differential bit encoding. This is due to the fact that known training sequences are periodically inserted for channel estimation, after which the phase drift is "reset" to 0 , providing the initial phase value. However, if square mQAM formats are considered, blind PNE algorithms can only estimate the phase wrapped in the interval $[-\pi / 4, \pi / 4]$. As a result, a phase tracking scheme is required for phase unwrapping to avoid the phase uncertainty issue. This can be done with a feedback loop (FL) or a digital phase tracking (DPT) algorithm implemented in a feed forward architecture.

\section{A. Feedback loop}

In CO-OFDM systems, due to the relatively long symbol duration, one symbol-delay feedback loop can be effectively applied for phase tracking as shown in Fig. 2 [12]. In this case, the laser phase noise is compensated in a two-stage algorithm. In the first stage, the received $m^{\text {th }}$ OFDM symbol is first rotated using the estimated CPE from the previous symbol as

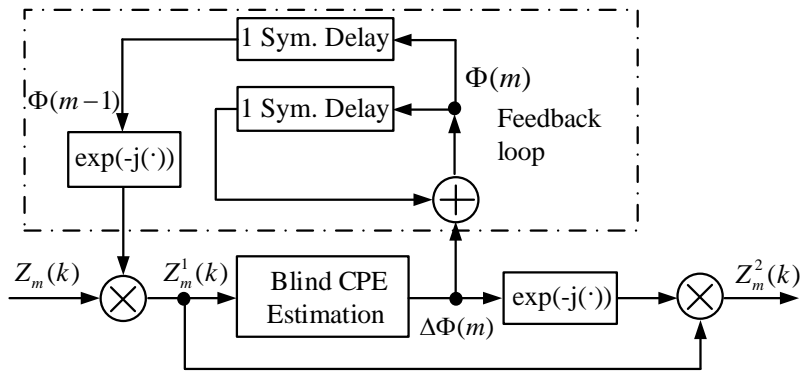

Fig. 2. Block diagram of blind PNE with a feedback loop for phase tracking

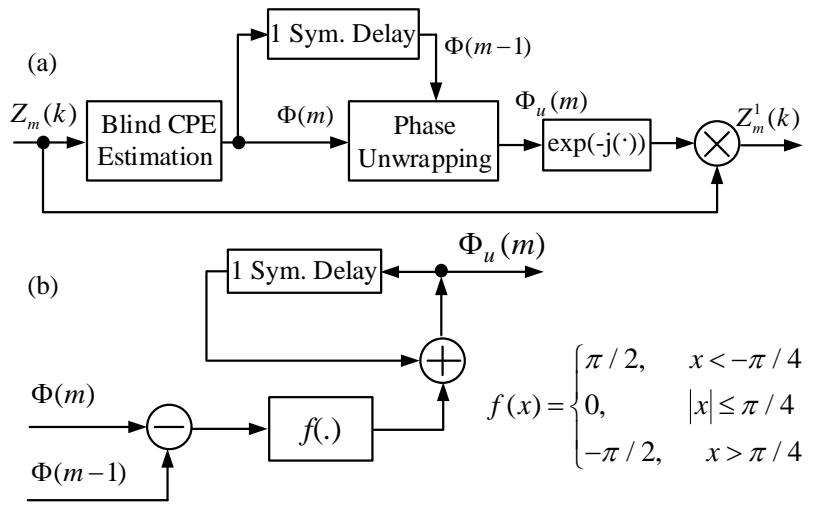

Fig. 3 (a) - block diagrams of (a) - blind PNE schemes without feedback loop, (b) - phase unwrapping block

$$
\begin{aligned}
Z_{m}^{1}(k) & =Z_{m}(k) \exp (-j \Phi(m-1)) \\
& =X_{m}(k) \exp (j \Delta \Phi(m))+\chi_{m}(k) \exp (-j \Phi(m-1))
\end{aligned}
$$

where $\Delta \Phi(m)$ is the residual CPE after equalization. Note that this equalization stage does not change the noise statistics and variance as $\chi_{m}(k)$ is Gaussian distributed. As a result, adding this equalization stage does not affect the performance of blind PNE algorithms. In the second stage of equalization, the residual CPE is estimated, compensated for and the phase is tracked as

$$
\Phi(m)=\Phi(m-1)+\Delta \Phi(m)
$$

Note that in this scheme, the phase can only be tracked if the residual CPE $\Delta \Phi(m)$ after equalization lines in the interval $[\pi / 4, \pi / 4]$. Otherwise, phase cycle slip occurs, which may lead to a catastrophic failure at the receiver.

\section{B. Digital Phase tracking}

The evolution of laser phase noise can also be tracked without a feedback loop using the following digital phase tracking algorithm

$$
\Phi_{u}(m)=\Phi(m)+\sum_{k=1}^{m} f(\Phi(k)-\Phi(k-1))
$$

where the function $f(x)$ keeps count of the phase cycles and is defined as 


$$
f(x)=\left\{\begin{array}{lr}
\pi / 2, & x<-\pi / 4 \\
0, & |x| \leq \pi / 4 \\
-\pi / 2, & x>\pi / 4
\end{array}\right.
$$

The general implementation block diagrams of blind PNE schemes without feedback loops and the phase unwrapping block are shown in the Fig. 3. This scheme employs only a single stage of compensation, and thus, can be much more computationally efficient in comparison to the previous scheme with FL. It can also be shown that the DPT algorithm (18) will fails if $\Phi(m)-\Phi(m-1)$ lines outside in the interval $[\pi / 4, \pi / 4]$.

\section{Cycle slip probability}

For mQAM formats, it has been discussed above that phase tracking algorithm may fail if the difference between the CPEs of the two neighbouring OFDM symbols $(\Phi(m)-\Phi(m-1))$ line outside the interval $[\pi / 4, \pi / 4]$, leading to phase cycle-slip.

Taking into account Eq. (15) we have

$$
\Phi(m)-\Phi(m-1)=\frac{1}{N} \sum_{n=0}^{N-1}\left(\phi_{m}(n)-\phi_{m-1}(n)\right)
$$

As the laser phase noise $\phi(t)$ is modelled as a Wiener process, $\phi_{m}(n)-\phi_{m-1}(n)$ can be modeled as a random Gaussian distributed variable with zero mean and a variance

$$
\Delta^{2}=2 \pi \beta T_{S}
$$

where $T_{\mathrm{S}}$ is the total OFDM symbol duration (including cyclic prefix). Therefore, $\Phi(m)$ - $\Phi(m-1)$, which is the mean value of $\phi_{m}(n)-\phi_{m-l}(n)$, is also a random Gaussian distributed variable with the same variance. As a result, the phase cycle-slip probability of blind PNE schemes can be calculated as

$$
\operatorname{Pr}=2 Q\left(\frac{\pi}{4 \Delta}\right)=2 Q\left(\frac{\pi}{4 \sqrt{2 \pi \beta T_{s}}}\right)
$$

where $Q$ is the $\mathrm{Q}$-function defined as

$$
Q(x)=\frac{1}{\sqrt{2 \pi}} \int_{x}^{\infty} \exp \left(-j z^{2} / 2\right) d z
$$

The cycle slip probability is shown in Fig. 4 as a function of the symbol duration linewidth product $\left(\beta T_{\mathrm{S}}\right)$. In CO-OFDM systems, when cycle slip occurs it causes all bits to be in error until the end of the OFDM frame, where the phase drift is reset due to training sequence. For single carrier transmission, an acceptable cycle slip probability (without requiring differential logical detection) might be $10^{-18}$ [19]. However, in CO-OFDM systems, because of training sequence, an acceptable cycle slip probability can be much higher. It has been shown in [7] that occurrence of cycle slip has little influence on the performance provided that is at least two order of magnitude less than the BER. As a result, for CO-OFDM system employing softdecision forward error correction, we conclude that an acceptable cycle slip probability might be $10^{-5}$. As shown in Fig. 4, the cycle slip probability of $10^{-15}$ occurs at $\beta T_{\mathrm{S}}=5 \times 10^{-3}$.

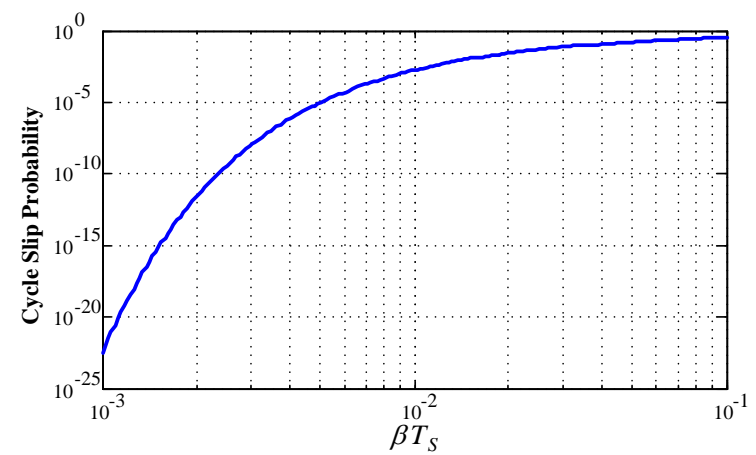

Fig. 4. Phase cycle-slip probability as a function of symbol-durationlinewidth product in CO-OFDM systems with blind PNE

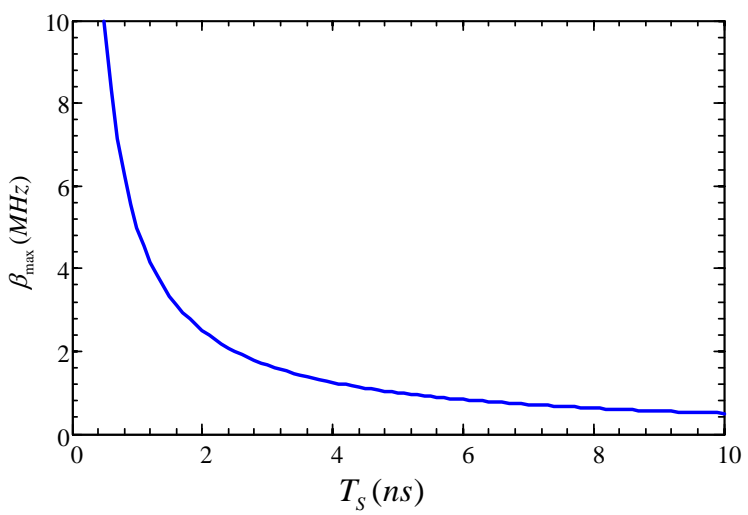

Fig. 5. Maximum allowable laser linewidth as a function of symbol duration for implementing blind PNE in CO-OFDM systems

This particularly highlights the challenge for implementing blind PNE techniques in CO-OFDM systems with long symbol duration. As shown in Fig. 5, when the OFDM symbol duration $T_{\mathrm{S}}=10 \mathrm{~ns}$, the laser linewidth $\beta$ must be below $500 \mathrm{kHz}$ in order to implement a fully blind PNE technique without differential bit encoding. However, for such systems, differential bit encoding and cycle slip can also be effectively avoided with quasi-blind PNE techniques where two bits are allocated in each OFDM symbol for phase tracking after blind PNE. This simple technique almost does not reduce the spectral efficiency and increase the complexity while significantly relaxing the requirements for transceivers' lasers. The linewidth requirement of quasi-blind PNE technique is out of scope of this paper and is open for future research.

\section{DECISION DIRECTED FREE BLIND PNE}

In this section we provide a detailed theoretical analysis of the decision-directed-free (DDF) blind PNE technique, which was proposed and experimentally demonstrated in [12]. This technique uses the following cost function, which is the mean value of the squared product of the projections of real and imaginary parts after rotation by a phase angle $\phi$

$$
J_{1}(\phi)=E\left\{\operatorname{Re}\left(Z_{m}(k) e^{-j \phi}\right)^{2} \cdot \operatorname{Im}\left(Z_{m}(k) e^{-j \phi}\right)^{2}\right\}_{k}
$$

where $E$ stands for the averaging operation over a portion or all of the subcarriers in the $m^{\text {th }}$ symbol.

For squared QAM formats (e.g. QPSK, 16QAM, 32QAM) with identical probabilities of constellation points, the 
proposed cost function reaches its maximum value at $\phi=\Phi(\mathrm{m})$ This phenomenon can be explained by the fact that ideal squared QAMs provide a "balance" between the real and imaginary parts of constellation points, thus, maximizing the mean value of the squared product of the projections of real and imaginary parts [12]. A similar cost function called dispersion minimization derotator (DDM), which is the dispersion of the projection of the constellation onto the real axis, was also considered in [20].

In a similar way with [20], the well-known stochastic gradient algorithm can be applied to maximize the cost function (24) as

$$
\begin{aligned}
& \phi_{k+1}=\phi_{k}+\mu E\left\{\operatorname{Re}\left(Z_{m}(k) e^{-j \phi}\right) \cdot \operatorname{Im}\left(Z_{m}(k) e^{-j \phi}\right)\right. \\
& \left.\times\left[\operatorname{Im}\left(Z_{m}(k) e^{-j \phi}\right)^{2}-\operatorname{Re}\left(Z_{m}(k) e^{-j \phi}\right)^{2}\right]\right\}
\end{aligned}
$$

which exploits the fact that

$$
\begin{aligned}
& (\partial / \partial \phi) \operatorname{Re}\left(Z_{m}(k) e^{-j \phi}\right)=\operatorname{Im}\left(Z_{m}(k) e^{-j \phi}\right) \\
& (\partial / \partial \phi) \operatorname{Im}\left(Z_{m}(k) e^{-j \phi}\right)=-\operatorname{Re}\left(Z_{m}(k) e^{-j \phi}\right)
\end{aligned}
$$

However, stochastic gradient algorithm usually requires tens of iterative steps upon convergence. In this case, the complexity is still an issue, especially in high-speed RGI CO-OFDM systems. Therefore, it is desirable to calculate the CPE without iterative algorithms. We will show that this is possible using the cost function (24).

Herein, we focus our analysis on square QAM formats. If the probabilities of constellation points are identical, which usually the case for modern transmission systems, it is easily to show that the following assumptions are valid [20].

\section{Assumptions}

i) The second cross-moment is separable, i. e.,

$$
E\left\{\operatorname{Re}\left(X_{m}(k)\right)^{2} \cdot \operatorname{Im}\left(X_{m}(k)\right)^{2}\right\}=E\left\{\operatorname{Re}\left(X_{m}(k)\right)^{2}\right\} E\left\{\operatorname{Im}\left(X_{m}(k)\right)^{2}\right\}
$$

ii) Most cross-moments vanish

$$
E\left\{\operatorname{Re}\left(X_{m}(k)\right)^{h} \cdot \operatorname{Im}\left(X_{m}(k)\right)^{v}\right\}=0
$$

if $h \neq 2, v \neq 2$ and $0<h, v \leq 3$

iii) The variances of the real and imaginary parts of the signal are the same

$$
E\left\{\operatorname{Re}\left(X_{m}(k)\right)^{2}\right\}=E\left\{\operatorname{Im}\left(X_{m}(k)\right)^{2}\right\}
$$

iv) $X_{m}(k)$ and $\chi_{m}(k)$ are statistically independent

Under these assumptions, straightforward calculations show that

$$
J_{1}(\phi)=\frac{P_{X}^{2}}{8} \cos (4 \phi-4 \Phi(m))+\frac{P_{X}^{2}}{8}+\frac{1}{2} P_{X} \sigma_{\chi}^{2}+\frac{1}{4} \sigma_{\chi}^{4}
$$

where $P_{X}^{2}$ is the signal power, and $\sigma_{\chi}^{2}$ is the variance of the noise term $\chi_{m}(k)$.

As a result, the cost function $J_{1}(\phi)$ can be written in this form
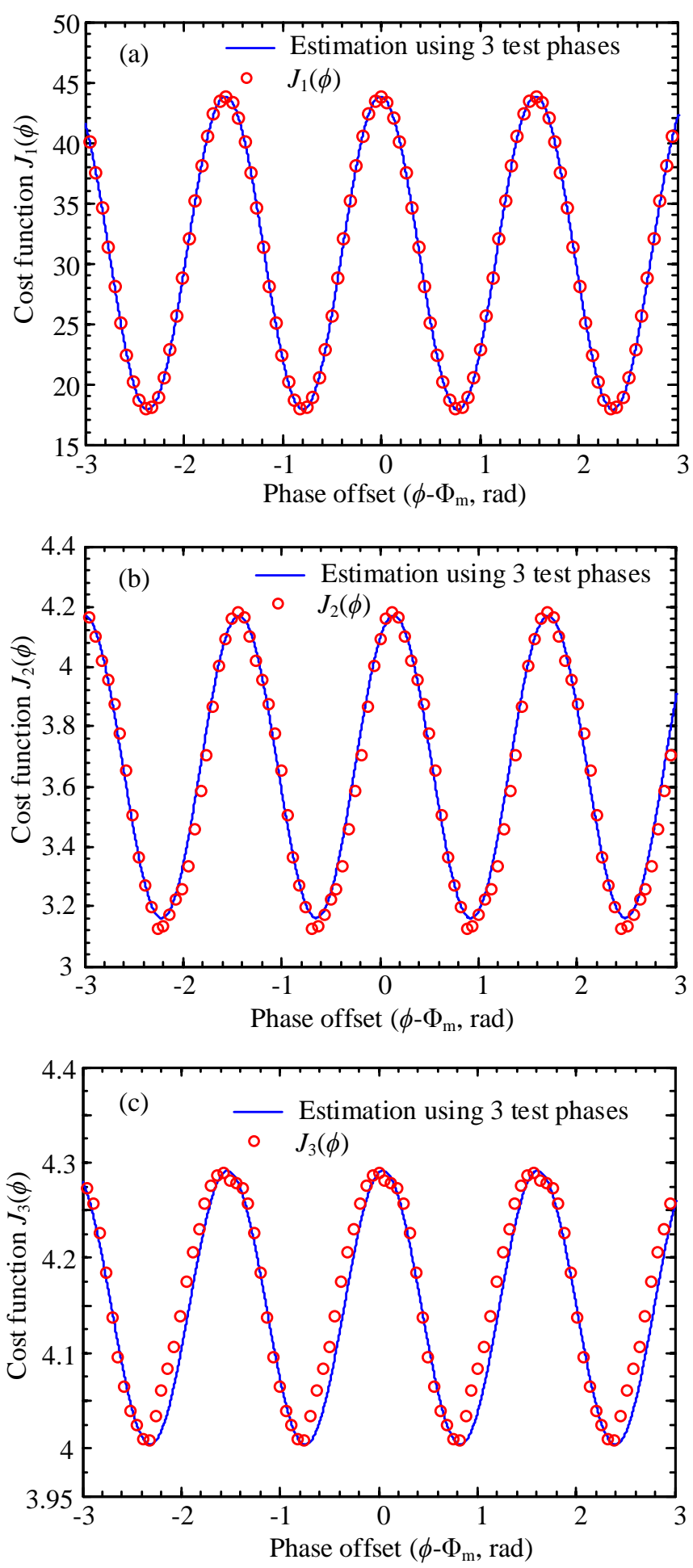

Fig. 6. Calculated cost functions $J_{1}(\phi), J_{2}(\phi)$ and $J_{3}(\phi)$ and its estimations using three test phases as function of phase offsets for 16QAM with $N=200$ subcarriers and $\mathrm{SNR}=6 \mathrm{~dB}$

$$
J_{1}(\phi)=A \cos (4 \phi-4 \Phi(m))+B,
$$

where $A, B, \Phi(m)$ are three variables to be determined. Knowing the form of the cost function, the CPE $(\Phi(m))$ can be easily defined using just three test phases, for example, $0, \pi / 4$ and $\pi / 8$ as follows 


$$
\begin{gathered}
B=\left(J_{1}(0)+J_{1}(\pi / 4)\right) / 2 \\
A=\sqrt{\left(J_{1}(0)-B\right)^{2}+\left(J_{1}(\pi / 8)-B\right)^{2}} \\
\Phi(m)=0.25 \cdot \operatorname{sgn}\left(B-J_{1}(\pi / 8)\right) \cdot \operatorname{acos}\left(\left(J_{1}(0)-B\right) / A\right),
\end{gathered}
$$

where $\operatorname{sgn}()$ is the sign function.

The calculated cost function $J_{1}(\phi)$ and its estimation using 3 test phases for 16-QAM with a SNR of $6 \mathrm{~dB}$ and a typical value of subcarrier $N=200$ are compared in Fig. 6(a), showing no mismatch. Similar results (not shown here) were obtained for QPSK, 32QAM and 64QAM. This confirms that the cost function $J_{1}(\phi)$ can be very well approximated using three parameters $A, B, \Phi(m)$ as shown in Eq. (28). As a consequence, with decision-directed-free (DDF) blind PNE technique, the CPE can be effectively calculated using only three test phases regardless of the modulation formats. This significantly reduces the implementation complexity in comparison with BPS, where 16-32 tests phases are required, depending on modulation formats.

To calculate the cost function $J_{1}(\phi), 3$ real multiplications per symbol are required. To further reduce the implementation complexity, we propose here two novel cost functions with similar properties

$$
\begin{aligned}
& J_{2}(\phi)=E\left\{\left|\operatorname{Re}\left(Z_{m}(k) e^{-j \phi}\right) \cdot \operatorname{Im}\left(Z_{m}(k) e^{-j \phi}\right)\right|\right\}_{k} \\
& J_{3}(\phi)=E\left\{\left|\operatorname{Re}\left(Z_{m}(k) e^{-j \phi}\right)\right|+\left|\operatorname{Im}\left(Z_{m}(k) e^{-j \phi}\right)\right|\right\}_{k}
\end{aligned}
$$

Unfortunately, there are no simple close-form expressions for the two cost functions $J_{2}(\phi)$ and $J_{3}(\phi)$. However, in a similar way to $J_{1}(\phi)$, the cost functions $J_{2}(\phi)$ and $J_{3}(\phi)$ reach their maximum values at $\phi=\Phi(m)$ for squared QAM formats. In addition, both cost functions $J_{2}(\phi)$ and $J_{3}(\phi)$ can be approximated well using the Eq. (28). As a result, the CPE can also be defined with the help of $J_{2}(\phi)$ and $J_{3}(\phi)$ using Eq. (2932 ) with reduced complexities. To calculate the cost function $J_{2}(\phi)$ only one real multiplication/symbol is required thus, reduces the complexity by 3 times in comparison to $J_{1}(\phi)$. Furthermore, in the case of $J_{3}(\phi)$, no multiplications are required, offering very low complexity in implementation. A comparison on the complexities of blind PNE techniques is given in section VI.

However, the complexity reductions associated with the use of the cost functions $J_{2}(\phi)$ and $J_{3}(\phi)$ also come with a price. As shown in Fig. 6(b)-(c) the deviations of the calculated and approximated functions for $J_{2}(\phi)$ and $J_{3}(\phi)$ can be observed. This mismatch can degrade the performance of DDF PNE techniques employing $J_{2}(\phi)$ and $J_{3}(\phi)$.

The root-mean-square-error (RMSE) of CPE as a function of SNR for DDF blind PNE techniques employing $J_{1}(\phi), J_{2}(\phi)$ and $J_{3}(\phi)$ for 16QAM CO-OFDM transmission with 100 subcarriers are presented in Fig. 7. Herein, the RMSE is calculated using Monte-Carlo simulation of the model (16) with 10000 runs. In Fig. 7, the best performance is achieved with $J_{1}(\phi)$, showing that a small RMSE of $0.1 \mathrm{rad}$ can be achieved at SNRs $>5.3 \mathrm{~dB}$. This result clearly indicates the

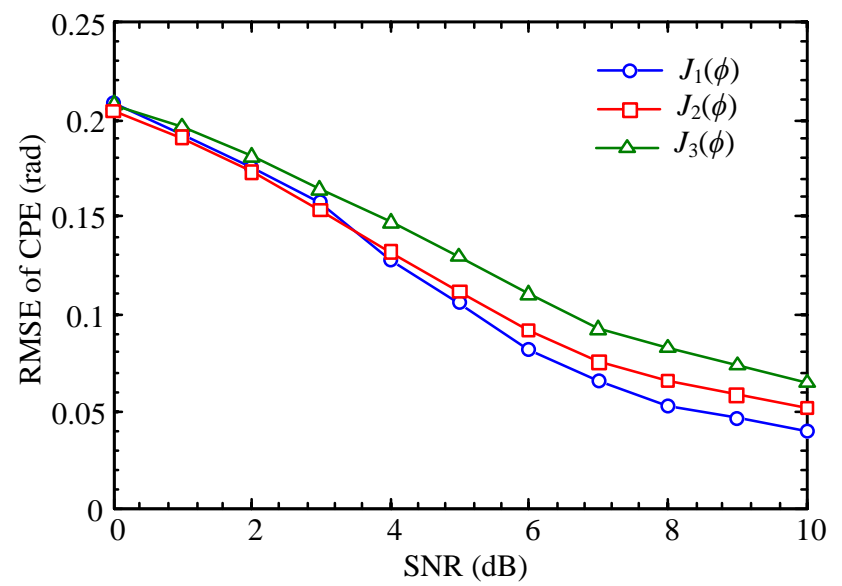

Fig. 7. Root-mean-square-error (RMSE) of CPE as a function of SNR for DDF PNE techniques employing $J_{1}(\phi), J_{2}(\phi)$ and $J_{3}(\phi)$ for 16QAM COOFDM transmission with $N=100$ subcarriers

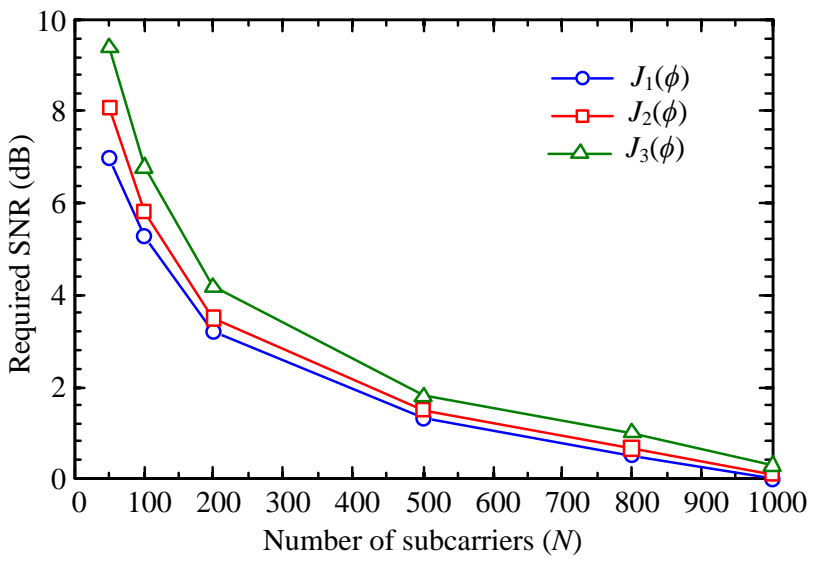

Fig. 8. Required SNRs for a RMSE of $0.1 \mathrm{rad}$ as a function of number of subcarriers $(N)$ for DDF PNE techniques employing $J_{1}(\phi), J_{2}(\phi)$ and $J_{3}(\phi)$ for 16QAM CO-OFDM transmission

high tolerance of the DDF blind PNE technique to Gaussian noise. However, when $J_{2}(\phi)$ and $J_{3}(\phi)$ are employed the required SNRs for a RMSE of $0.1 \mathrm{rad}$ are $5.8 \mathrm{~dB}(0.5 \mathrm{~dB}$ SNR penalty) and $6.8 \mathrm{~dB}$ (1.5 dB SNR penalty), respectively. However, the SNR penalties associated with the uses of $J_{2}(\phi)$ and $J_{3}(\phi)$ reduce if the number of subcarrier $N$ is increased. As shown in Fig. 8, at a low value of $N$ of 50, the SNR penalties of $J_{2}(\phi)$ and $J_{3}(\phi)$ are $1.1 \mathrm{~dB}$ and $2.4 \mathrm{~dB}$, respectively. However, if $N=1000$, SNR penalties of $J_{2}(\phi)$ and $J_{3}(\phi)$ are reduced to only $0.1 \mathrm{~dB}$ and $0.3 \mathrm{~dB}$, respectively. This result indicates that if the number of OFDM subcarrier is large, $J_{2}(\phi)$ and $J_{3}(\phi)$ can be used efficiently instead of $J_{1}(\phi)$ to offer a very low complexity blind PNE.

\section{PERFORMANCE COMPARISON}

In this section, through numerical simulation, we compare the performance of DDF blind PNE technique with difference cost functions with BPS and pilot-aided techniques. We focus our discussion on high order modulation formats, namely 16QAM and 64QAM. It has been shown experimentally in [12] that DD-based blind PNE technique performs poorly for such high modulation formats. As a result, we do not take into account DD blind PNE in our comparison in this paper. 

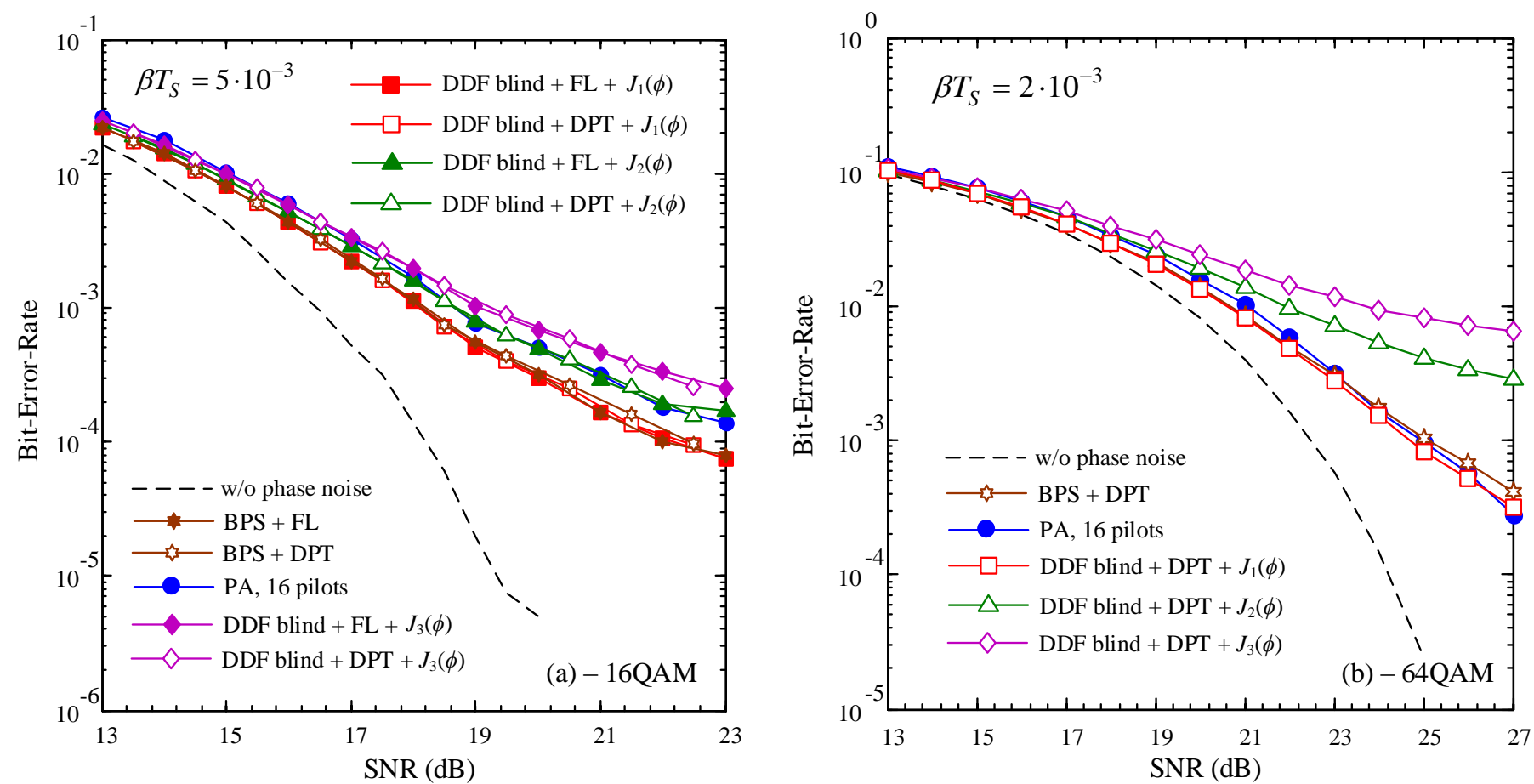

Fig. 9(a) -BER performances of PNE techniques, including PA-aided with 16 pilots (blue), BPS with 16 test phases (brown) and DDF blind PNE with different cost functions (red- $J_{1}(\phi)$, green- $J_{2}(\phi)$, pink- $J_{3}(\phi)$ ) with feedback loop (solid) and digital phase tracking (open) for 16QAM, the symbol duration linewidth product is $5 \cdot 10^{-3}$; (b) - BER performance of the same PNE (only showing digital phase tracking) for 64QAM, the symbol duration linewidth product is $2 \cdot 10^{-3}$.
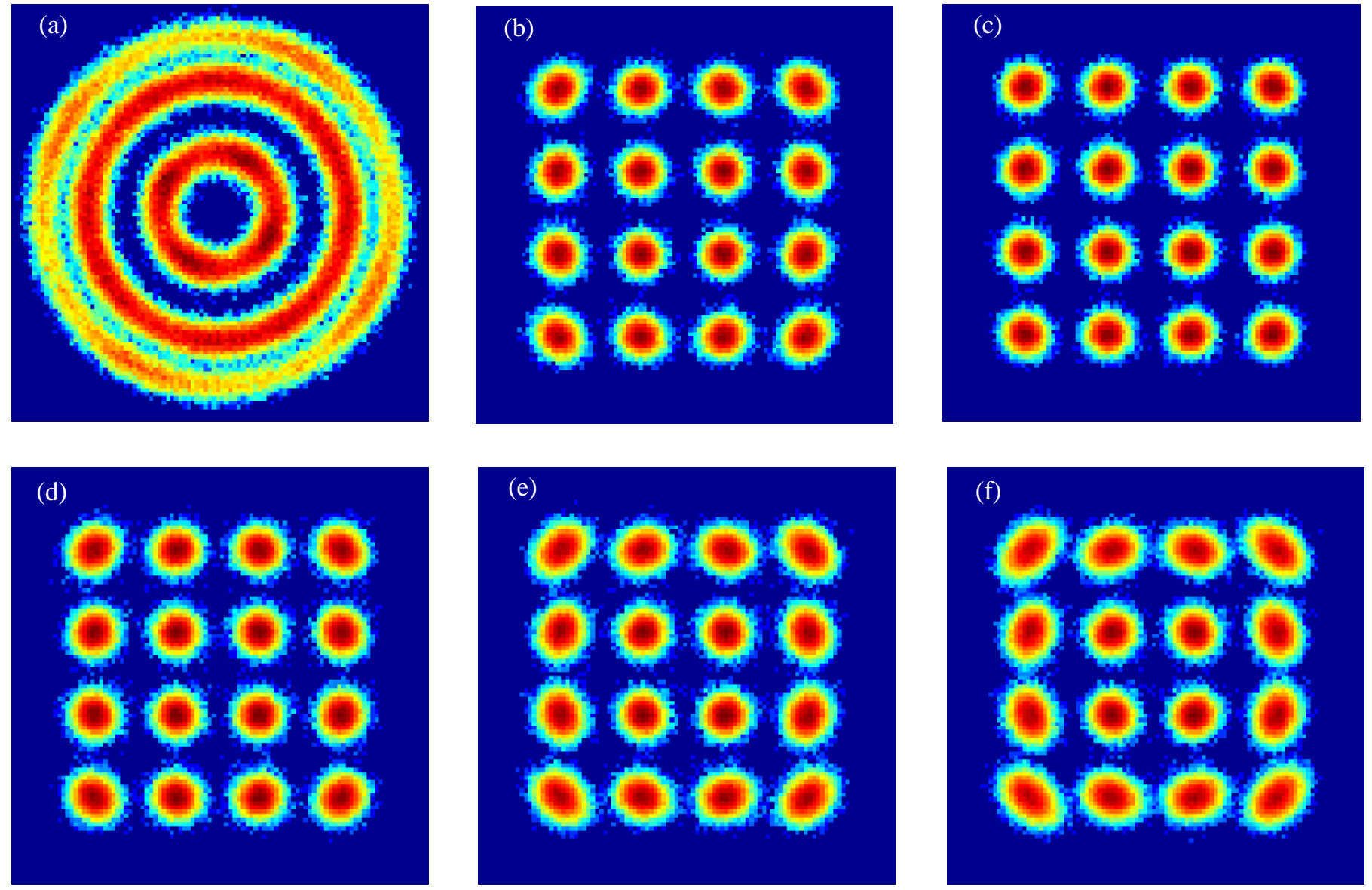

Fig. 10: Constellation diagrams for 16QAM at a SNR of $23 \mathrm{~dB}$, before PNE (a), after PNE using PA-aided technique with 16 pilots (b), after PNE with DDF blind PNE technique with digital phase tracking and $J_{1}(\phi)$ (c), after PNE using BPS with 16 test phases (d), after PNE DDF blind PNE technique with digital phase tracking and $J_{2}(\phi)$ and $J_{3}(\phi)(\mathrm{e}, \mathrm{f})$. 

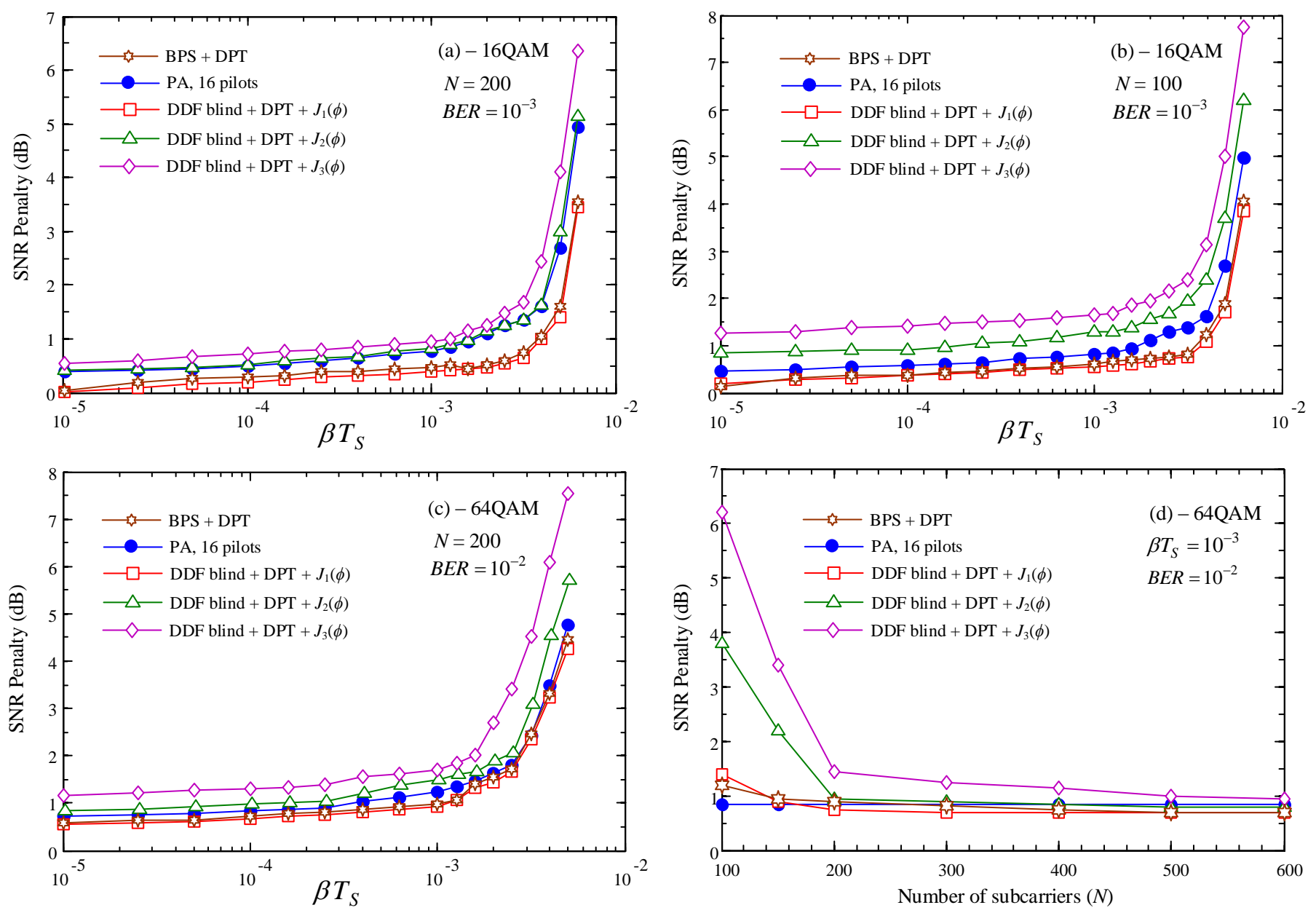

Fig. 11 (a) - The SNR penalty at a BER of $10^{-3}$ as a function of $\beta T_{\mathrm{S}}$ for PA-aided, BPS and DDF blind PNE with different cost functions for $16 \mathrm{QAM}$ transmission with 200 subcarriers; (b) - similar result for 16QAM and 100 subcarriers; (c) - similar result for 64QAM at a BER of $10^{-2}$ and 200 subcarriers; (d) - SNR penalty as a function of $N$ at a BER of $10^{-2}$ for 64QAM and $\beta T_{\mathrm{S}}$ of $10^{-3}$.

For investigation the performance of blind PNE techniques for CO-OFDM systems, there are two critical parameters, namely the number of OFDM subcarriers $N$ and the symbol duration linewidth product $\beta T_{s}$. In our simulation, we vary $\beta T_{s}$ by varying the laser linewidth while the OFDM symbol duration is kept constant at $10 \mathrm{~ns}$, which is equivalent to a subcarrier spacing of $100 \mathrm{MHz}$. The system BER is evaluated through direct error counting using Monte Carlo simulation with a total number of symbols of $2 \cdot 10^{5}\left(8 \cdot 10^{5}, 1.2 \cdot 10^{6}\right.$ bits for 16QAM and 64QAM, respectively). In the light of (16), we take into account here only the back-to-back transmission regime for simplicity to study the tolerance of PNE techniques to AWGN and laser linewidth. The simulation results for 16QAM and 64QAM are presented in Fig. 9-11.

In Fig. 9(a), the BER performances of PNE techniques, including PA-aided with 16 pilots, BPS with 16 test phases and DDF blind with FL, DPT and different cost functions are compared for $\beta T_{s}=5 \cdot 10^{-3}$ and $N=200$. It can be seen that FL and DPT offer the same performance in all considered blind PNE techniques. Taking into account the fact that DPT is much more computationally efficient for practical implementations, we will further consider only DPT. In Fig. 9(a), DDF blind with the cost function $J_{1}(\phi)$ shows the same performance in comparison to the complicated BPS with
16 test phases. This confirms the high performance of DDF blind PNE technique. Even though the complexity of DDF blind with $J_{1}(\phi)$ is relatively low in comparison to BPS, different cost functions, namely $J_{2}(\phi)$ and $J_{3}(\phi)$ can also be effectively applied to reduce further the complexity. In Fig. 9(a), DDF blind with $J_{2}(\phi)$ shows a similar performance in comparison to PA-aided with 16 pilots. On the other hand, the implementation of DDF blind with $J_{3}(\phi)$ leads to $\sim 1 \mathrm{~dB}$ SNR penalty at a BER level of $10^{-3}$.

Similar results for 64QAM for $\beta T_{s}=2 \cdot 10^{-3}$ is shown in Fig. 9 (b). Here DDF with $J_{1}(\phi)$ also offers a similar performance in comparison to BPS. However, for 64QAM, the effectiveness of blind PNE technique is reduced as DDF blind with $J_{1}(\phi)$ and BPS does not offer significant performance advantage over PA-aided with 16 pilots. However, blind PNE techniques are still attractive here because of the high spectral efficiency offered. The constellation diagrams for 16QAM at SNR = $23 \mathrm{~dB}$ before and after phase compensation with different PNE techniques are shown in Fig. 10.

The power penalties at a BER of $10^{-3}$ as functions of $\beta T_{\mathrm{S}}$ for PA-aided, BPS and DDF blind PNE with different cost functions for 16QAM transmission with 200 subcarriers are shown in Fig. 11(a). At $1 \mathrm{~dB}$ SNR penalty, the linewidth tolerance of BPS and DDF blind with $J_{1}(\phi)$ is $\beta T_{s}=4 \cdot 10^{-3}$. For 
DDF blind with $J_{2}(\phi)$ and $J_{3}(\phi)$ the linewidth tolerances are $\beta T_{s}$ $=2 \cdot 10^{-3}$ and $10^{-3}$, respectively.

When the number of OFDM subcarrier is reduced to 100, DDF blind with $J_{1}(\phi)$ still shows excellent performance, with a similar linewidth tolerance to the case of 200 subcarriers. However, the performances of DDF blind with $J_{2}(\phi)$ and $J_{3}(\phi)$ degrade significantly. At $1 \mathrm{~dB}$ SNR penalty, the linewidth tolerance of DDF blind with $J_{2}(\phi)$ in this case is $\beta T_{s}=5 \cdot 10^{-4}$ while $1 \mathrm{~dB}$ SNR penalty even cannot be achieved with DDF blind with $J_{3}(\phi)$ for $\beta T_{s}>10^{-5}$. This indicates that DDF blind with $J_{3}(\phi)$ is not suitable for a low value of $N$.

Similar results for 64QAM at a BER of $10^{-2}$ and $N=200$ are shown in Fig. 11(c). At $1 \mathrm{~dB}$ SNR penalty, the linewidth tolerance of DDF blind with $J_{1}(\phi)$ in this case is $\beta T_{s}=10^{-3}$. The SNR penalties as functions of $N$ at a BER of $10^{-2}$ for 64QAM and $\beta T_{\mathrm{S}}$ of $10^{-3}$ are shown in Fig. 11(d). It should be noted, unlike other blind PNE techniques, the performance of PA-aided technique is independent of $N$. Blind phase search and DDF blind with $J_{1}(\phi)$ show excellent performance when $N>100$. On the other hand, DDF blind with $J_{2}(\phi)$ and $J_{3}(\phi)$ should be considered only if $N$ is equal or bigger than 200 .

\section{COMPLEXITY COMPARISON}

Herein, we compare the complexities BPS and DDF blind PNE techniques and in term of the required number of real multipliers, adders, comparators and decisions. The rootsquare operation is counted as one multiplier. The result is shown in the Table I, where $M$ is the number of test phases in BPS and $N_{1} \leq N$ is the number of subcarriers used for PNE. The required real multipliers for DDF blind PNE with $J_{1}(\phi)$ and $J_{2}(\phi)$ are around 7 and 21 times less than those of BPS with 16 test phases. The numbers of adders are also reduced by 10 times. In addition, DDF blind PNE does not require any comparators and decisions. This clearly indicates that DDF blind PNE is much more computational efficient than BPS.

TABLE I

COMPLEXITIES OF BSP AND DDF BLIND PNE TECHNIQUES

\begin{tabular}{lcccc}
\hline PNE & Multipliers & Adders & Comparators & Decisions \\
\hline BPS & $4 N_{l} M$ & $2 N_{l} M$ & $M+1$ & $N_{l} M+N_{l}$ \\
DDF $+J_{1}(\phi)$ & $9 N_{l}+4$ & $3 N_{l}+4$ & 0 & 0 \\
DDF $+J_{2}(\phi)$ & $3 N_{l}+4$ & $3 N_{l}+4$ & 0 & 0 \\
DDF $+J_{3}(\phi)$ & 4 & $3 N_{l}+4$ & 0 & 0 \\
\hline
\end{tabular}

\section{CONCLUSION}

In this paper, we have shown that blind PNE can be effectively applied for CO-OFDM transmissions with BPS and DDF blind PNE. Using only three test phases, DDF blind PNE technique can offer a comparable performance in comparison with BPS with 16 tests phases, and thus, offering an effective solution for practical implementation. In addition, when the number of subcarriers is sufficient $(N>200)$ the complexity of DDF blind can be further significantly reduced with two novel proposed cost functions.

\section{ACKNOWLEDGMENT}

This work was partially supported by the UK EPSRC programme Grants UNLOC and PEACE (EP/J017582/1 and EP/L000091/1) and the European Commission's 7th Framework Programme FP/2007-2013 grant 318415 (FOX-C).

\section{REFERENCES}

[1] F. Buchali, R. Dischler, and X. Liu, "Optical OFDM: A promising highspeed optical transport technology," Bell Labs Technical Journal, vol. 14, pp. 125-146, 2009.

[2] S. Wu and Y. Bar-Ness, "OFDM Systems in the Presence of Phase Noise: Consequences and Solutions," IEEE Transactions on Communications, vol. 52, pp. 855-855, 2004.

[3] L. Xiang, S. Chandrasekhar, Z. Benyuan, P. J. Winzer, A. H. Gnauck, and D. W. Peckham, "448-Gb/s Reduced-Guard-Interval CO-OFDM Transmission Over $2000 \mathrm{~km}$ of Ultra-Large-Area Fiber and Five 80GHz-Grid ROADMs," JLT, vol. 29, pp. 483-490, 2011.

[4] W. Shieh, "Maximum-Likelihood Phase and Channel Estimation for Coherent Optical OFDM," PTL, IEEE, vol. 20, pp. 605-607, 2008.

[5] S. T. Le, T. Kanesan, M. McCarthy, E. Giacoumidis, I. Phillips, M. F. Stephens, et al., "Experimental Demonstration of Data-dependent Pilotaided Phase Noise Estimation for CO-OFDM," OFC, San Francisco, California, 2014, p. Tu3G.4.

[6] S. T. Le, T. Kanesan, E. Giacoumids, N. Doran, and A. Ellis, "Quasipilot Aided Phase Noise Estimation for Coherent Optical OFDM Systems," Photonics Technology Letters, IEEE, vol. 26, pp. 504-507, 2014.

[7] Z. Qunbi, M. H. Morsy-Osman, and D. V. Plant, "Low Overhead IntraSymbol Carrier Phase Recovery for Reduced-Guard-Interval COOFDM," JLT, vol. 31, pp. 1158-1169, 2013.

[8] M. E. Mousa-Pasandi and D. V. Plant, "Zero-overhead phase noise compensation via decision-directed phase equalizer for coherent optical OFDM," Optics Express, vol. 18, pp. 20651-20660, 2010/09/27 2010.

[9] H. Youngsun and C. Wonzoo, "Non-Data-Aided Phase Noise Suppression Scheme for CO-OFDM Systems," Photonics Technology Letters, IEEE, vol. 25, pp. 1703-1706, 2013.

[10] T. Bo, L. Huang, and C.-K. Chan, "Image Processing Based Common Phase Estimation for Coherent Optical Orthogonal Frequency Division Multiplexing System," OFC, Los Angeles, California, 2015, p. W1E.8.

[11] T. Pfau, S. Hoffmann, and R. Noe, "Hardware-Efficient Coherent Digital Receiver Concept With Feedforward Carrier Recovery for MQAM Constellations," JLT, vol. 27, pp. 989-999, 2009.

[12] S. T. Le, M. McCarthy, N. Mac Suibhne, P. A. Haigh, E. Giacoumidis, N. Doran, et al., "Decision-Directed-Free Blind Phase Noise Estimation for CO-OFDM," in Optical Fiber Communication Conference, Los Angeles, California, 2015, p. W1E.5.

[13] L. Tomba, "On the effect of Wiener phase noise in OFDM systems," Communications, IEEE Transactions on, vol. 46, pp. 580-583, 1998.

[14] T. Pollet, M. Van Bladel, and M. Moeneclaey, "BER sensitivity of OFDM systems to carrier frequency offset and Wiener phase noise," Communications, IEEE Transactions on, vol. 43, pp. 191-193, 1995.

[15] Y. Mostofi, D. C. Cox, and A. Bahai, "ICI mitigation for mobile OFDM receivers," in Communications, 2003. ICC '03. IEEE International Conference on, 2003, pp. 3351-3355 vol.5.

[16] A. F. Molisch, M. Toeltsch, and S. Vermani, "Iterative Methods for Cancellation of Intercarrier Interference in OFDM Systems," Vehicular Technology, IEEE Transactions on, vol. 56, pp. 2158-2167, 2007.

[17] L. Myung-Kyu, L. Seung-Chan, and Y. Kyeongcheol, "Blind Compensation for Phase Noise in OFDM Systems over Constant Modulus Modulation," IEEE Transactions on Communications, vol. 60, pp. 620-625, 2012

[18] T. Kanesan, S. T. Le, D. Roque, and A. D. Ellis, "Non-rectangular perfect reconstruction pulse shaping based ICI reduction in COOFDM," Optics Express, vol. 22, pp. 1749-1759, 2014/01/27 2014.

[19] M. G. Taylor, "Phase Estimation Methods for Optical Coherent Detection Using Digital Signal Processing," JLT, vol. 27, pp. 901-914, 2009

[20] C. Wonzoo, W. A. Sethares, and C. R. Johnson, "Performance analysis of blind adaptive phase offset correction based on dispersion minimization," IEEE Transactions on Signal Processing, vol. 52, pp. 1750-1759, 2004. 